\title{
The simulation and research of the workflow modeling based on Petri nets
}

\author{
Meng Guang-Xue ${ }^{1, a}$, Guo-Hong ${ }^{1, b}$, Wu-Jing ${ }^{1, c}$ \\ Department of Computer.North China Institute of Science and Technology,Beijing 101601,China \\ 1a339521662@QQ.COM
}

keywords: Workflow model, Petri nets, Research, Simulation

Abstract: It is very important for reasonable analysis of complex workflow model based on Petri nets. Based on the four basic models, the complex workflow model is simplified by equivalent layer. According to the equal servicing time formula of series model, parallel model and selection model, calculate the simplified the model and build simulation models. Analyze its reasonableness through the simulation and theoretical calculation

Petri net model is primarily a tool to establish the relationship between the properties through the workflow task description, it has the advantage to be able to fully concurrent, discrete event attribute describes the dynamic aspects, through the use of a graphical description of the property of tools for modeling, combined with theoretical and mathematical definition, analysis and evaluation model algorithm aspects typical Petri nets have the following characteristics: first, it is a graphical modeling tool with an intuitive, easy to use the advantage, in the resource constraints or resource conflicts can concurrently visualize and describe resolve; secondly, it has the ability to describe mathematical modeling, information theory and stochastic process theory combined with the system uncertainty or randomness analysis.

Activities in the process of implementation, depending on the execution order, the basic model defines four implementation process [1]:

(1) the order of execution is also referred to as a serial execution: Figure 1 (a), a plurality of sub-order of tasks sequentially executed. If task A and B two tasks to perform, A task execution over again perform $B$ tasks.

(2) Select the execution order of execution is also known as a conditional branch: Figure 1 (c), first performed to judge the conditions, which determine the conditions set up on the implementation of the tasks performed which task, in accordance with the conditions of the priority the level of selective execution.

(3) may also be referred to simultaneously perform parallel execution sequence: Figure 1 (b), the two tasks performed simultaneously or in any order, such as task $\mathrm{C}$ and task B is executed in parallel.

(4) Repeat the cycle can also be called the order of execution: Figure 1 (d), each task as long as the condition is true, can be recycled repeatedly executed.

(a)

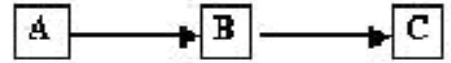

(b)

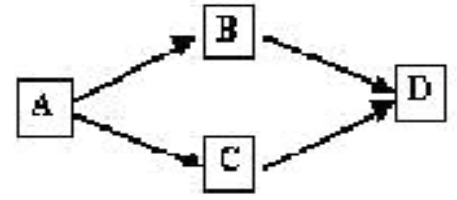

(a)

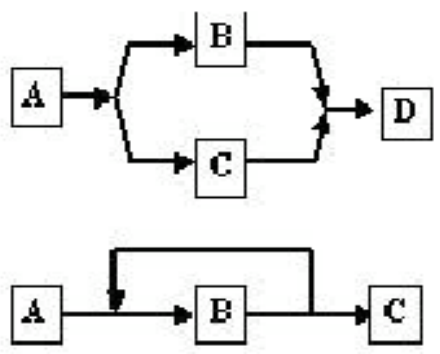

Figure 1 Four basic model of Workflow 


\section{Equivalent Business Hours workflow model analysis method}

For a complex Petri net workflow model based on direct equivalent service time analysis more difficult, treatment must be based on the basic model is simplified, calculated separately for each kind of connection equivalent service time, finally in the calculation of performance parameters of the overall model.

1、Parallel model equivalence analysis service time [4]

Performance expectations for equivalent service hours:

$$
\begin{aligned}
E(y) & =\int_{0}^{\infty} y f_{B}(y) d y \\
& =\sum_{i=1}^{k+1} \frac{1}{\lambda_{i}}-\sum_{i=1}^{k} \sum_{j=i+1}^{k+1} \frac{1}{\lambda_{i}+\lambda_{j}}+\sum_{i=1}^{k-1} \sum_{j=i+1 l=j+1}^{k} \sum_{i}^{k+1} \frac{1}{\lambda_{i}+\lambda_{j}+\lambda_{l}}+\mathrm{L}+(-1)^{k} \frac{1}{\sum_{i}^{k+1} \lambda_{i}}
\end{aligned}
$$

2、 Equivalent Analysis Methods of service time model [4]

If you were to obey $\hat{t_{1}}, \hat{t_{2}}, \mathrm{~L} \hat{t_{n}}$ random variable parameters $\lambda_{1}, \lambda_{2} \ldots \lambda_{n}$ exponential distribution function, then the equivalent service time performance expectations are expressed as:

$$
E_{B_{n}}(y)=\sum_{i=1}^{n} \alpha_{i} \frac{1}{\lambda_{i}}
$$

3、 Equivalent series model analysis service time [4]

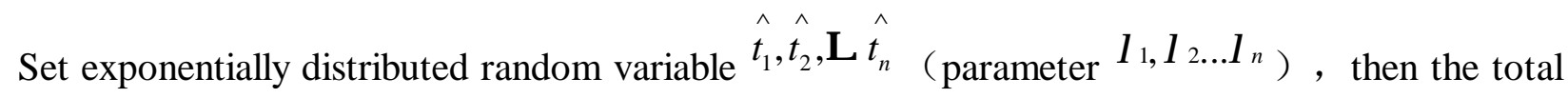
service time equivalent to the $n$ modules:

$$
\frac{1}{\lambda}=\sum_{i=1}^{n} \frac{1}{\lambda_{i}}
$$

The following sales process as an example to illustrate the specific performance-based workflow modeling and Petri net analysis.

\section{Model of}

First, the user needs to relate here the sales process, including:

1. Users place orders to the company, pointed out the need of a computer configuration and the number of users after the company received orders to check stock and price information to make decisions based on the configuration user requests.Model of.

2. To carry out the above orders inspections and audits by the audit is acceptable, then the next step, otherwise the notice or letter to the user informed of the results or suggest modifications, modify the order, and then handed over to the clerk to issue feedback.

3. On the one hand you want to notify the user after receiving payment orders, on the other hand the production of notice to be issued. These two processes are parallel processes. After issuing the notice to be assembled computer production and delivery process ends at this time. Were analyzed according to the needs of the flowchart drawn throughout the sales process shown in Figure 2.

4. The entire process through the following activities to complete the sale process operations.

(1) Users place orders to the company, pointed out the need for product configuration and quantity, Petri model expressed by the changes $t 0$.

(2) The company receives customer orders, changes in the model with $t 1$, said.

(3) Firm orders for the user to check, meaning the following three events concurrent process, an immediate transition is available, said: calculate the price, with changes in the model represents $\mathrm{t} 2$; 
check whether parts inventory to meet demand, with changes in the model represents $\mathrm{t} 3$; configure checks, verify customer orders technically feasible, with changes in the model represents $t 4$.

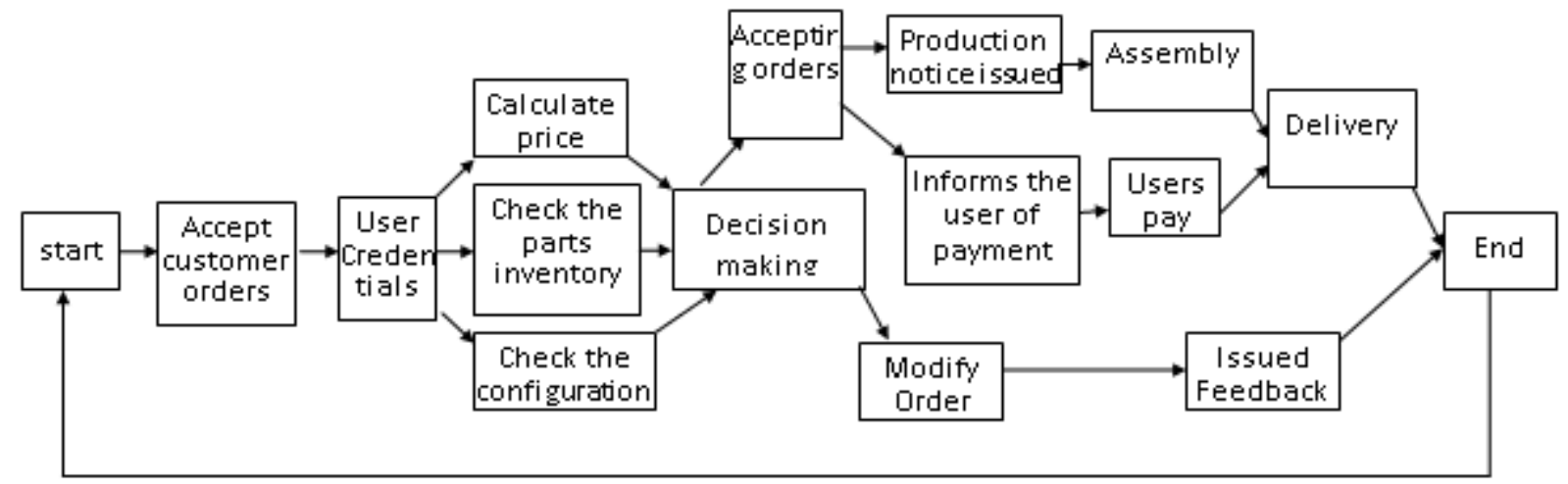

Figure 2 Product sales process flow chart

(4) Decision-making, with changes in the model represents t5. If, then continue by checking; if you do not pass inspection, the user is issued a letter explaining why the order can not be completed, and proposed amendments, modify the order, with changes in the model t10 said then handed over to the clerk to issue feedback in model expressed by changes $t 11$.

(5) Ready to receive the order confirmation notice, which means on the one hand to inform the user of payment, on the other hand issued a notice of two parallel processes of production, which itself may represent an immediate transition. Notify the user of payment, with changes in the model represents $t 8$.

(6) User notice, make a payment, with changes in the model represents $t 9$.

(7) Issued a notice of production, with changes in the model represents t6.

(8) Assembled computer, with changes in the model represents $t 7$.

(9) Delivery, with changes in the model represents t12.

For system flow chart examples above, in the definition of Petri net model, has the relationship between the various tasks with the library node $p(O)$ to represent; $t$ rectangle $(\square)$ represents the task with the changes in the system nodes; task and work associated with flow logic expressed arc $(\curvearrowright$ ) that the implementation of a state with a black dot in the circle $(\odot$ ) tag. Thus the implementation of the workflow process modeling used in the change of the meaning as defined in Table 1 below.

Table 1 Product sales process workflow Petri net model changes the meaning of

\begin{tabular}{|c|c|c|c|}
\hline Change & The change of meaning & Change & The change of meaning \\
\hline$t_{1}$ & Accept customer orders & $t_{2}$ & Calculation of price \\
\hline$t_{3}$ & Check the parts inventory & $t_{4}$ & Check the configuration \\
\hline$t_{5}$ & Decision making & $t_{6}$ & Production notice issued \\
\hline$t_{7}$ & Assembly & $t_{8}$ & Informs the user of payment \\
\hline$t_{9}$ & Users pay & $t_{10}$ & Modify Order \\
\hline$t_{11}$ & Issued Feedback & $t_{12}$ & Delivery \\
\hline
\end{tabular}

Petri net model based on the analysis system flow chart 2, combined with the establishment of the system is defined in Table 1 of the workflow shown in Figure 3. When users submit orders to the company, the first to accept the company's audit and inspection, inspection and audit process to concurrent activities, the use of Petri net model is described; assuming the probability of accepting orders for, modify the order of probability using an instantaneous changes to represent; the company orders submitted after inspection by the time the order is ready to accept an immediate transition can also be used, concurrency issues production payment notification and notify the user that the two processes. 


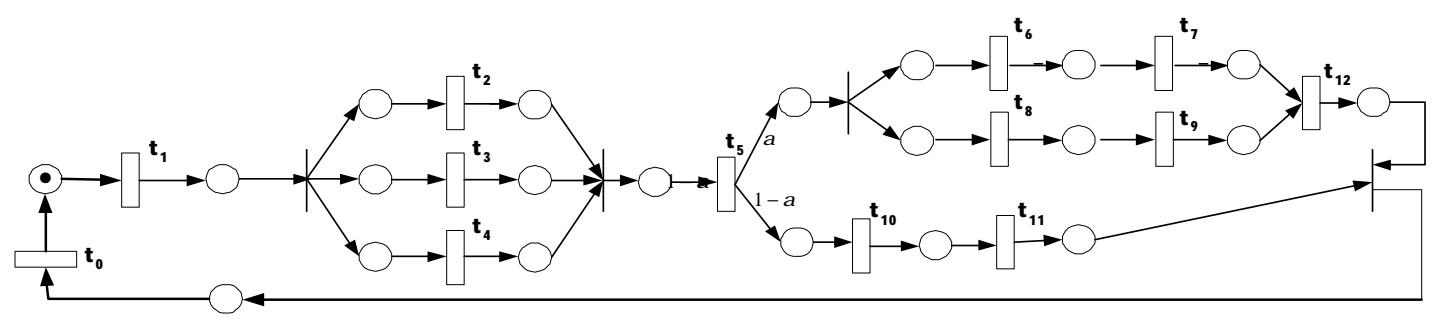

Figure 3 Product sales process workflow net model diagram

\section{Performance analysis of the equivalent model}

1、 The average delay time petri nets model of Change

In this paper, the process of changes in assumptions $t_{0}, t_{1}, t_{2}, t_{3}, t_{4}, t_{5}, t_{6}, t_{7}, t_{8}$, $t_{9}, t_{10}, t_{11}, t_{12}$ the average delay time was:

$$
\begin{aligned}
& \frac{1}{\lambda_{0}}=38, \frac{1}{\lambda_{1}}=15, \frac{1}{\lambda_{2}}=8, \frac{1}{\lambda_{3}}=30, \frac{1}{\lambda_{4}}=20, \frac{1}{\lambda_{5}}=40, \frac{1}{\lambda_{6}}=5, \frac{1}{\lambda_{7}}=120, \frac{1}{\lambda_{8}}=40, \\
& \frac{1}{\lambda_{9}}=2880, \frac{1}{\lambda_{10}}=5, \frac{1}{\lambda_{11}}=40, \frac{1}{\lambda_{12}}=150
\end{aligned}
$$

Wherein the delay time in minutes. In addition, the $t_{5}$ branch office is located to receive orders selection probability $\alpha_{1}=0.91$.

2、Equivalent performance computing

Performance computing equivalence principle is: first from the inside out, through changes in performance equivalent to the simple to the complex structure of the easiest in the simplified model of the process, should be based on four basic models, the first analytical model series, parallel basic structure, selection and loop relationship, and then calculates the overall system according to the relevant performance parameters corresponding equivalent formulas. After the string, and simplification and selection, to obtain the model shown in FIG. 4:

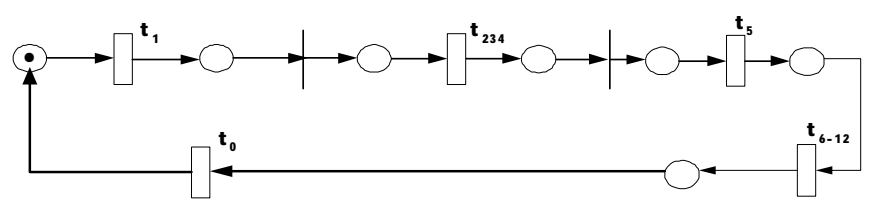

Figure 4 final simplified sales process model diagram

3. Finally, by the series formula (3) evaluate the total performance of the equivalent time:

$$
\begin{gathered}
\frac{1}{\lambda_{123456789101112}}=\frac{1}{\lambda_{0}}+\frac{1}{\lambda_{1}}+\frac{1}{\lambda_{234}}+\frac{1}{\lambda_{5}}+\frac{1}{\lambda_{6789101112}} \\
=38+15+38.77+40+2911.507=3043.277
\end{gathered}
$$

After the calculated performance equivalent time model, the number of tasks can be calculated performance parameters such as throughput and average processing system according to the appropriate formula. Examples of the system there is only one mark model, so the throughput is equivalent to the reciprocal of the time, That $\lambda_{123456789101112}=3.29 \times 10^{-4}$ (task / per minute), the average number of day processing tasks for $24 \times 60 \times 3.29 \times 10^{-4}=0.474$ ( Months)。(The computer simulation part slightly) 


\section{Conclusion}

Complex workflow model to analyze performance is necessary, based on the model established by the actual sales process is very complex and enormous size, the performance of the analysis more difficult, must be complex workflow model based on four basic models equivalent simplified calculation method based on the quantitative analysis of the basic model, provide a reasonable basis for improving and perfecting models.

Hebei Engineering Technology Research Center for IOT Data acquisition \& Processing

\section{References}

[1] Francesco Basile,Pasquale Chiacchio,Domenico Teta. A hybrid model for real time simulation of urban traffic[J]. Control Engineering Practice . 2011 (2)

[2] Xiao Liang, Zhi li Liu,Kun Qian. Capacity Analysis of Signalized Intersections under Mixed Traffic Conditions[J]. Journal of Transportation Systems Engineering and Information Technology . 2011 (2)

[3] Andrews M, Anta A F, Zhang L, Zhao Wenbo. Routing forenergy minimization in the speed scaling model . Proceedingsof the 29th IEEE Conference on Computer Communications(INFOCOM'10)。2010，339-345

[4]Jean-Pierre Signoret,Yves Dutuit,Pierre-Joseph Cacheux,Cyrille Folleau,Stéphane Collas,Philippe Thomas. Make your Petri nets understandable: Reliability Block Diagrams driven Petri nets.[J]. Reliability Engineering and System Safety . 2012

[5]Pang Shanchen, Li Yin, He Hua, Lin Chuang. A model fordynamic business processes and process changes. ChineseJournal of Electronics, 2011, 20(4): 632-636

[6] LiangbingFeng,MasanaoObayashi,TakashiKuremoto,KunikazuKobayashi. A learning Fuzzy Petri net model[J]. IEEJ Trans Elec Electron Eng . 2012 (3)

[7] W.M.P.vanderAalst.Three Good reasons for Usinga Petri-net-based Workflow management System[J], In:NavatheS, WakayamaT, eds.Proeeedings of the intermational Working Conferenee on Infonnation and Proeess Integrtion in Enterprises(IPIC'96), Camebridge, Massachusetts: Kluwer Aeademic Publishers, Nov1996:179-201.

[8] WFMC.Wbrkflow Management Coalition temunology \& glossary(WFMC1000)[M],1994.

[9] WFMC.The workfiow reference model(WFMC1003) [M],1995.

[10] Guo Feng, Wei Guang Deng misty A service-oriented Petri net model and structural operational semantics [J]. Small micro-computer system. 2013 (12) 